Pacific Journal of Mathematics

INVARIANT SUBSPACES OF NONSELFADJOINT CROSSED
PRODUCTS 


\title{
INVARIANT SUBSPACES OF NON-SELFADJOINT CROSSED PRODUCTS
}

\author{
MiChaEL MCASEY
}

Let $\mathfrak{R}$ be the von Neumann algebra crossed product determined by a maximal abelian selfadjoint algebra $L^{\infty}(X)$ and an automorphism of $L^{\infty}(X)$. The algebra $\mathfrak{L}$ is generated by a bilateral shift $L_{\delta}$ and an abelian algebra $\mathfrak{M}_{L}$ isomorphic to $L^{\infty}$; the non-selfadjoint subalgebra $\mathfrak{L}_{+}$of $\mathfrak{Q}$ is defined to be the weakly closed algebra generated by $L_{\delta}$ and $\mathfrak{M}_{L}$. The commutant of $\mathcal{L}$ is the algebra $\Re$, also a crossed product. The invariant subspace structure of $\Omega_{+}$is investigated. It is shown that full, pure invariant subspaces for $\mathfrak{L}_{+}$are unitarily equivalent by a unitary operator in $\mathfrak{R}$ if and only if their associated projections are equivalent in $\mathfrak{M}_{L}^{\prime}$. Furthermore, a multiplicity function can be associated with each invariant subspace. The algebra $\Re$ contains a subalgebra $\Re_{+}$analogous to $\mathfrak{R}_{+}$. It is shown that subspaces invariant for both algebras $\mathfrak{L}_{+}$and $\mathfrak{R}_{+}$can be parameterized in terms of certain subsets of the cartesian product $\mathrm{Z} \times X$.

1. Introduction. In their fundamental paper of 1936, Murray and von Neumann used the algebraic idea of a crossed product to exhibit special types of von Neumann algebras. The result of this construction is a crossed product of an abelian von Neumann algebra with a group of automorphisms of the algebra. This crossed product is now commonly called a group-measure algebra. Today, the study of crossed products in operator theory has become important not only for the examples it yields but also for its contribution to the general structure theory of operator algebras. For example, they have been useful in unraveling the structure of type III von Neumann algebras [11]. Indeed, Feldman and Moore [4] have recently shown that it is likely that every von Neumann algebra can be realized as a crossed product-perhaps of a complicated nature.

In this paper we shall consider crossed products of the type first considered by Murray and von Neumann [7]. We shall concentrate our attention on certain non-selfadjoint subalgebras of their crossed products, subalgebras which we call non-selfadjoint crossed products. These subalgebras stand in the same relation to group-measure algebras as $H^{\circ}$ (the space of boundary values of bounded analytic functions on the unit disc) stands in relation to $L^{\infty}$ of the circle. Two specific representations of the non-selfadjoint crossed products will be useful; these are defined in $\S 2$ and are 
denoted by $\mathfrak{Z}_{+}$and $\Re_{+}$. In addition some background is given and results discussed which may be helpful in viewing the present paper in its proper perspective. In $\S 3$ we show that certain equivalence classes of invariant subspaces of the algebra $\mathfrak{L}_{+}$can be identified with equivalence classes of projections. Furthermore, with each class of invariant subspaces we associate an essentially unique multiplicity function. In $\S 4$ it is shown that subspaces invariant under both $\mathfrak{S}_{+}$and $\mathfrak{R}_{+}$have a particularly simple form. These subspaces can be used to provide a perspicuous set of canonical models for the $\mathbb{R}_{+}$-invariant subspaces in certain instances. After an example in $\S 5$, we conclude with remarks which relate the algebra $\mathfrak{L}_{+}$to crossed products defined elsewhere in the literature.

2. Definitions and preliminaries. The construction of the crossed product of a von Neumann algebra $2 \mathfrak{C}$ with an automorphism $\alpha$ can be found in many places in the literature (eg. [3, $\S$ I. 9.2], $[6, \S 3])$. We shall consider two specific representations of the crossed product for the case that $\mathfrak{A}$ is $L^{\infty}(X)$, a maximal abelian selfadjoint algebra (m.a.s.a.) and $\alpha$ is given by a transformation $\tau$ of $X$.

Let $X$ be a $\sigma$-finite measure space with positive measure $\mu$. All such spaces are assumed to be standard Borel. Let $\tau$ be an invertible measurable transformation and assume that the measures $\mu$ and $\mu \circ \tau$ are mutually absolutely continuous; i.e., $\mu$ is quasi-invariant under $\tau$. Using the product of counting measure on the integers, $Z$, and the measure $\mu$ on $X$, we may realize $Z \times X$ as a measure space. The Lebesgue space $L^{2}(\boldsymbol{Z} \times X)$ is also a Hilbert space with inner product

$$
(f, g)=\sum_{n=-\infty}^{\infty} \int_{X} f(n, x) \overline{g(n, x)} d \mu(x) \quad\left(f, g \in L^{2}(\boldsymbol{Z} \times X)\right) .
$$

When no confusion results, we shall often abbreviate $L^{2}(\boldsymbol{Z} \times X)$ to $L^{2}$.

For any integer $n$, let $J_{n}$ denote the Radon-Nikodym derivative $J_{n}=d \mu \circ \tau^{-n} / d \mu$. We define the following operators on $L^{2}(\boldsymbol{Z} \times X)$ : for $f$ in $L^{2}(Z \times X)$ and $\varphi$ in $L^{\infty}(X)$, let

$$
\begin{aligned}
& \left(L_{\hat{o}} f\right)(n, x)=J_{1}^{1 / 2}(x) f\left(n-1, \tau^{-1} x\right) \\
& \left(R_{\hat{\delta}} f\right)(n, x)=f(n-1, x) \\
& \left(L_{\varphi} f\right)(n, x)=\varphi(x) f(n, x) \\
& \left(R_{\varphi} f\right)(n, x)=\varphi\left(\tau^{-n} x\right) f(n, x),
\end{aligned}
$$

and

$$
(J f)(n, x)=J_{n}^{1 / 2}(x) f\left(-n, \tau^{-n} x\right) .
$$


All of the operators are bounded, $L_{\hat{o}}$ and $R_{\delta}$ are unitary operators, and $J$ is an anti-unitary operator (i.e., $(J f, J g)=(g, f)$ ) (cf. [8, Lemma 3.6.1]). Let $\mathfrak{M}_{L}$ (respectively $\mathfrak{M}_{R}$ ) denote the algebra generated by $\left\{L_{\varphi} \mid \varphi \in L^{\infty}(X)\right\}$ (resp. $\left\{R_{\varphi} \mid \varphi \in L^{\infty}(X)\right\}$ ). The left (resp. right) von Neumann algebra crossed product of $L^{\infty}(X)$ with (the automorphism implemented by) $\tau$ is defined to be the von Neumann algebra $\mathfrak{R}$ (resp. $\mathfrak{R}$ ) generated by $\mathfrak{M}_{L}$ and $L_{\hat{o}}$ (resp. $\mathfrak{M}_{R}$ and $R_{\delta}$ ). In this paper we shall be concerned with particular non-selfadjoint subalgebras of $\&$ and $\Re$ of the type first considered by Arveson [1]. Define the left (resp. right) non-selfadjoint crossed product to be the weakly closed algebra $\mathfrak{L}_{+}$(resp. $\mathfrak{R}_{+}$) generated by $\mathfrak{M}_{L}$ and $L_{\grave{\delta}}$ (resp. $\mathfrak{M}_{R}$ and $R_{\delta}$ ). Note in particular that while $L_{\delta}$ is in both $\&$ and $\mathfrak{L}_{+}, L_{\delta}^{*}$ lies in $\mathbb{Q}$ but not in $\mathfrak{S}_{+}$.

It is well known that $\mathbb{S}^{\prime}$, the commutant of $\mathbb{R}$, equals $\Re$ and also that $\Re^{\prime}=\mathbb{R}$ (cf. $\S 3.6$ of [8]). The operator $J$ is an involution on $L^{2}$ and has the property that $J \Omega J=\Re$. As a consequence of this last equation we will prove theorems for the left-algebra only and leave the corresponding theorems about $\Re$ to the reader.

Although $\mathfrak{M}_{L}$ is abelian, $\mathbb{Z}$ is not abelian since $L_{\hat{o}}$ does not commute with $\mathfrak{M}_{L}$. We do have, however, the following vestige of commutativity: $L_{\hat{\delta}} L_{\varphi}=L_{\varphi_{\circ} \tau}-1 L_{\hat{j}}$. Writing this equation as $L_{\hat{o}} L_{\varphi} L_{\hat{\delta}}^{*}=$ $L_{\varphi_{0 \tau}-1}$ shows that the unitary operator $L_{\delta}$ normalizes $\mathfrak{M}_{L}$; i.e., $L_{i} \mathfrak{M}_{L} L_{\delta}^{*}=\mathfrak{M}_{L}$. Another consequence of this observation is that we may realize $\mathbb{R}$ (resp. $\mathbb{L}_{+}$) as the weak closure of the set of operators of the form $\sum_{n=-k}^{k} L_{\varphi_{n}} L_{\delta}^{n}$ (resp. $\sum_{n=0}^{k} L_{\varphi_{n}} L_{i}^{n}$ ). The proofs of these statements and the corresponding statements for the algebra $\mathfrak{R}$ are elementary computations and are omitted.

In this paper we concentrate on the invariant subspaces for the algebras $\mathfrak{L}_{+}$and $\Re_{+}$. To place our results in perspective we note that the algebra $\mathfrak{L}_{+}$can be constructed by using algebras $\mathfrak{M}_{L}$ which are not isomorphic to $L^{\infty}(X)$. For example in case $\mathfrak{M}_{L}$ is isomorphic to a factor (satisfying certain technical hypotheses), the invariant subspaces for $\mathfrak{L}_{+}$and $\Re_{+}$have been completely classified in our paper written with Muhly and Saito and have a particularly elegant form. Theorem 3.3 in [6] asserts that the (pure) invariant subspaces for $\mathfrak{L}_{+}$are parameterized in essentially the same way as the Beurling-Lax-Halmos theorem characterizes the invariant subspaces of the shift. We shall show that the algebras $\mathbb{L}_{+}$for which $\mathfrak{M}_{L}$ is a m.a.s.a. as considered here produce a very different nonselfadjoint algebra than the one defined in [6]; one striking difference is that the analogue of Beurling's theorem fails. Nevertheless, the analysis in both cases is based on the fact that $L_{\delta}$ is a bilateral shift.

The first step in our attack is to show that particular unitary 
equivalence classes of (pure) invariant subspaces of $\mathbb{S}_{+}$can be identified with equivalence classes of projections in $\mathfrak{M}_{L}^{\prime}$. This observation "reduces" the problem to investigating these latter equivalence classes. It should be noted that this step is motivated by the Beurling-Lax-Halmos theorem, but does not solve the problem in a concrete fashion. It develops that it is natural to make a separate analysis of the subspaces which are invariant under both $\mathbb{S}_{+}$and $\Re_{+}$. (The weakly closed algebra generated by $\mathfrak{L}_{+}$and $\Re_{+}$is denoted by $\mathfrak{Q}_{+} \vee \Re_{+}$.) In case $\tau$ is an ergodic but not a periodic transformation on $X$, the subspaces invariant under $\mathfrak{L}_{+} \vee \Re_{+}$can be parameterized quite nicely by certain subsets of $Z \times X$. For the case that $\tau$ is periodic, not every $\mathfrak{S}_{+} \vee \Re_{+}$-invariant subspace is determined by a subset of $Z \times X$ (cf. $\S 5$ ) but those that are sufficiently numerous that the analysis may proceed with them alone. The next task is to determine when two $\mathfrak{S}_{+} \vee \Re_{+}$-invariant subspaces are unitarily equivalent by a unitary in $\Re$, the commutant of $\mathbb{L}$. To do this we associate with each $\mathfrak{Q}_{+}$-invariant subspace a "multiplicity function" which provides a connection between an invariant subspace and its corresponding subset in $\boldsymbol{Z} \times X$. It will be shown that two invariant subspaces are unitarily equivalent by a unitary operator in $\Omega$ if and only if the associated multiplicity functions are identical.

We conclude this section by recording terminology used for subspaces of $L^{2}$. A (closed) subspace $\mathscr{C}^{\mathrm{C}}$ of $L^{2}(\boldsymbol{Z} \times X)$ is: leftinvariant (or $\mathbb{S}_{+}$-invariant) if $\mathbb{S}_{+} \mathscr{C} \subseteq \mathscr{C}$; left-reducing if $\mathbb{L}_{\mathscr{L}} \subseteq$ $\mathscr{C}$; left-pure if $\mathscr{C}$ is left-invariant and contains no (nonzero) leftreducing subspace; and left-full if the smallest left-reducing subspace containing $\mathscr{C}$ is $L^{2}$. A subspace $\mathscr{C}$ is said to be right-invariant (or $\Re_{+}$-invariant) in case $\Re_{+} \mathscr{C} \subseteq \mathscr{C}$. The definitions of the other "right-" terms are similar. We say a subspace is left-right-invariant or two-sided invariant or $\mathfrak{S}_{+} \vee \Re_{+}$-invariant in case it is invariant for both $\mathfrak{L}_{+}$and $\Re_{+}$(and hence for the algebra $\mathfrak{L}_{+} \vee \Re_{+}$).

3. Equivalence classes of left-invariant subspaces. The major theorem of this section shows that the equivalence classes of invariant subspaces for the algebra $\mathbb{L}_{+}$can be identified with certain equivalence classes of projections. Moreover, each such equivalence class can be identified in terms of a multiplicity function. The following proposition shows that the analysis of the invariant subspace structure of $\mathfrak{S}_{+}$may be reduced, in part, to known results about the invariant subspaces of $L_{\hat{j}}$. The proposition can be found in [6].

Proposition 3.1. Let $\mathscr{M}$ be a left-invariant subspace in 
$L^{2}(Z \times X)$. Then

(1) $\mathscr{L}$ reduces $\mathfrak{M}_{L}$;

(2) $\mathscr{L}$ reduces $\&$ if and only if $\mathscr{C}$ reduces $L_{\dot{\sigma}}$;

(3) $\mathscr{C}$ is pure if and only if $\bigcap_{n \geq 0} L_{\delta}^{n} \mathscr{C l}=\{0\}$; and

(4) $\mathscr{C l}$ is full if and only if $\mathbf{V}_{n \leq 0} L_{\dot{o}}^{n} \mathscr{M}=L^{2}(\boldsymbol{Z} \times X)$.

Recall that a subspace $\mathscr{C}$ is said to be wandering for a unitary operator $U$ in case $U^{m} \mathscr{C} \perp U^{n} \mathscr{C}$ for any two distinct integers $n$ and $m$. The following elementary lemma about $\mathfrak{L}_{+}$-invariant subspaces will be useful in the sequel. An analogous statement is valid for the algebra $\Re_{+}$.

LEMMA 3.2. If $\mathscr{C}$ is an $\mathbb{S}_{+}$-invariant subspace then $\mathscr{F}=\mathscr{C} \Theta$ $L_{\delta} \mathscr{C l}$ is a wandering subspace. Moreover, the projection $P_{\mathscr{S}}$ onto $\mathscr{F}$ lies in $\mathfrak{M}_{L}^{\prime}$.

Proof. Since $L_{\delta}$ is a unitary operator, the first statement is immediate. To show that the projection $P_{\mathscr{F}}$ is in $\mathfrak{M}_{L}^{\prime}$, we need to use the fact that $L_{\delta}$ normalizes $\mathfrak{M}_{L}$. Observe first that since the subspace $\mathscr{C}$ is invariant for the algebra $\mathfrak{R}_{+}$, it reduces $\mathfrak{M}_{L}$ by the preceding proposition. In addition, $L_{i} \mathscr{C l}$ is invariant under $\mathfrak{M}_{L}$ since

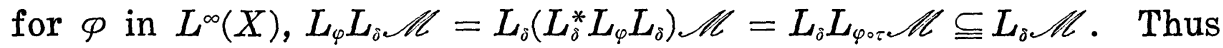
$L_{\hat{\delta}} \mathscr{C l}$ and, of course, $\left(L_{\delta} \mathscr{L C}\right)^{\perp}$ reduce $\mathscr{A}_{L}$. Hence $\mathscr{F}=\mathscr{H} \cap\left(L_{\hat{o}} \mathscr{C l}\right)^{\perp}$ reduces $\mathfrak{M}_{L}$ and so $P_{\mathscr{S}}$ lies in $\mathfrak{M}_{L}^{\prime}$.

Let $\mathscr{C}$ be any left-reducing subspace. Then $\mathscr{H}$ is invariant (and hence reducing) for the full von Neumann algebra $\&$ and so the projection $P_{\mathscr{N}}$ onto $\mathscr{C}$ lies in $\mathbb{Q}^{\prime}=\Re$. Thus any reducing subspace for $\Omega_{+}$is simply the range of a projection in $\Re$. It is for this reason and because of the following well known result that we shall be concerned mainly with the pure invariant subspaces of $\Omega_{+}$.

Proposition 3.3. Let $\mathscr{C l}$ be an $\mathbb{S}_{+}$-invariant subspace. Then $\mathscr{C}=\mathscr{C}_{1} \oplus \mathscr{C}_{2}$ where $\mathscr{L}_{1}$ is a left-reducing subspace and $\mathscr{L}_{2}$ is a left-pure subspace.

Proof. Set $\mathscr{A}_{1}=\bigcap_{n \geq 0} L_{\dot{\delta}}^{n} \mathscr{C l}$ and $\mathscr{C}_{2}=\mathscr{C} \ominus \mathscr{C}_{1}$. Since $L_{\hat{o}}$ normalizes $\mathfrak{M}_{L}, \mathfrak{M}_{L}$ leaves $L_{\hat{\delta}}^{n} \mathscr{C l}$ invariant for all $n$. Hence $\mathfrak{M}_{L}$ leaves $\mathscr{C}_{1}=\bigcap_{n \geq 0} L_{\delta}^{n} \mathscr{C}$ invariant. Since $\mathscr{M}_{1}$ reduces $L_{\hat{o}}, \mathscr{M}_{1}$ reduces $\mathfrak{S}_{+}$by Proposition 3.1. Since $\mathfrak{M}_{L}$ leaves $\mathscr{L}_{2}=\mathscr{C} \ominus \mathscr{C}_{1}$ invariant and since $L_{i} \mid \mathscr{C}_{2}$ is pure, $\mathscr{L}_{2}$ is a left-pure invariant subspace by Proposition 3.1 again.

The next objective is to identify the relevant equivalence classes 
of certain invariant subspaces for the algebra $\mathbb{L}_{+}$. Let $P$ and $Q$ be projections in a von Neumann algebra $\mathfrak{A}$. We say $P$ is equivalent to $Q$ in $\mathfrak{A}$ (or relative to $\mathfrak{A}$ ) in case there is a partial isometry $V$ in $\mathfrak{A}$ such that $V^{*} V=P$ and $V V^{*}=Q$. This relationship is denoted by $P \sim Q$. There is also a pre-order on the projections with respect to a von Neumann algebra $\mathfrak{A}$. A projection $P$ is dominated by a projection $Q$ in $\mathfrak{A}(P \leqq Q$ in $\mathfrak{U})$ in case there is a projection $E$ in $\mathfrak{A}$ such that $P \sim E$ in $\mathfrak{A}$ and $E \leqq Q$.

We continue our preparatory remarks by indicating several different ways to view the Hilbert space and the algebras with which we shall be concerned. First note that $L^{2}(Z \times X)$ may be identified with the Hilbert space direct sum of a countable number of copies of $L^{2}(X), \sum_{-\infty}^{\infty} \oplus L^{2}(X)$. Dixmier [3, pp. 23-24] shows how we may identify this space as the Hilbert space tensor product $\ell^{2}(\boldsymbol{Z}) \otimes L^{2}(X)$. In addition, Dixmier (p. 153) also shows how we may identify this tensor product with the direct integral $\int_{X}^{\oplus} \mathscr{H}(x) d x$ where $\mathscr{C}(x)$ is simply $\ell^{2}(\boldsymbol{Z})$ for each $x$; we shall write this as $\int_{X}^{\oplus} \ell^{2}(\boldsymbol{Z}) d x$. In a similar way we may identify the algebra $\mathfrak{M}_{L}$ with the von Neumann algebra tensor product $\boldsymbol{C}_{\ell^{2}(\boldsymbol{Z})} \otimes L^{\infty}(X)$ where $C_{\ell^{2}(\boldsymbol{Z})}^{\prime}$ denotes the algebra of scalar multiples of the identity acting on the space $\ell^{2}(\boldsymbol{Z})$ and $L^{\infty}(X)$ is, of course, the algebra of multiplication operators on $L^{2}(X)$. This identification maps the operator $L_{\varphi}$ in $\mathfrak{M}_{L}$ to the operator $I \otimes M_{\varphi}$ where $M_{\varphi}$ is the multiplication operator determined by $\varphi$ in $L^{\infty}(X)$ acting on $L^{2}(X)$. From this we are able to identify the commutant of $\mathfrak{M}_{L}: \mathfrak{M}_{L}^{\prime}=\left(\boldsymbol{C}_{\ell^{2}(\boldsymbol{Z})} \otimes L^{\infty}(X)\right)^{\prime}=$ $\boldsymbol{C}_{\iota^{2}(\boldsymbol{Z})}^{\prime} \otimes L^{\infty}(X)^{\prime}=\mathscr{L}\left(\ell^{2}(\boldsymbol{Z})\right) \otimes L^{\infty}(X)$, where $\mathscr{L}\left(\ell^{2}(\boldsymbol{Z})\right)$ is the full algebra of operators on $\ell^{2}(\boldsymbol{Z})$. Finally we may identify this last tensor product with the direct integral $\int_{X}^{\oplus} \mathscr{L}\left(\ell^{2}(\boldsymbol{Z})\right) d x$.

The last new concept we shall need is that of a multiplicity function for a subspace. Let $\mathscr{C}$ be a left-invariant subspace with wandering subspace $\mathscr{F}=\mathscr{C} \ominus L_{i} \mathscr{C l}$. From Lemma 3.2, we know that the projection $P_{\mathscr{F}}$ onto the space $\mathscr{F}$ lies in $\mathfrak{M}_{L}^{\prime}$. By the preceding discussion we may write $P_{\mathscr{F}}=\int_{X}^{\oplus} P(x) d x$ where $P(x)$ is a projection in $\mathscr{L}\left(\ell^{2}(\boldsymbol{Z})\right)$ for almost all $x$. The multiplicity function of the subspace $\mathscr{C}$ is the function $m$ defined by the equation

$$
m(x)=\text { dimension of range of } P(x)=\text { rank } P(x) .
$$

This notion of multiplicity will allow us to analyze completely the $\mathscr{L}_{+} \vee \Re_{+}$-invariant subspaces in several concrete situations.

In the following two theorems, we will be concerned with the 
algebra $\mathfrak{Q}_{+}$and so the terms "invariant", "pure", "full", etc. are all assumed to be "left-invariant", etc.

THEOREM 3.4. For $i=1,2$ let $\mathscr{M}_{i}$ be a pure invariant subspace for the algebra $\mathfrak{L}_{+}$. Let $\mathscr{F}_{i}=\mathscr{L}_{i} \ominus L_{\delta} \mathscr{L}_{i}$ be the associated wandering subspace with corresponding projection $P_{\mathscr{F}_{i}}$ and multiplicity function $m_{i}$. The following statements are equivalent.

(1) $P_{\mathscr{M}_{1}}=R_{v} P_{\mathbb{N}_{2}} R_{v}^{*}$ for a partial isometry $R_{v}$ in $\Re$, so that $\mathscr{M}_{1}=R_{v} \mathscr{L}_{2}$

(2) The multiplicity function for $\mathscr{M}_{2}$ dominates the multiplicity function for $\mathscr{C}_{1}$ almost everywhere; i.e., $m_{1}(x) \leqq m_{2}(x)$ (a.e.); and

(3) $P_{\mathscr{F}_{1}} \leqq P_{\mathscr{F}_{2}}$ in $\mathfrak{M}_{L}^{\prime}$.

Proof. We first prove that the first statement implies the second. As noted above we may identify $\mathfrak{M}_{L}^{\prime}$ with $\int_{X}^{\oplus} \mathscr{L}\left(\ell^{2}(\boldsymbol{Z})\right) d x$ and so we may write $R_{v}=\int_{X}^{\oplus} v(x) d x$, where $v(x)$ is a partial isometry (a.e.) in $\mathscr{L}\left(\ell^{2}(\boldsymbol{Z})\right)$. In addition, since $P_{\mathscr{F}_{i}}$ is in $\mathfrak{M}_{L}^{\prime}$, we may write $P_{\mathscr{F}_{i}}=\int_{X}^{\oplus} P_{i}(x) d x$ where $P_{i}(x)$ is a projection (a.e.). Now observe that since $P_{\mathscr{N}_{1}}=R_{v} P_{\mathscr{M}_{2}} R_{v}^{*}$ we have $P_{\mathscr{F}_{1}}=P_{\mathbb{N}_{1}}-L_{\hat{\delta}} P_{\mathscr{N}_{1}} L_{\hat{\delta}}^{*}=R_{v} P_{\mathbb{N}_{2}} R_{v}^{*}-$ $L_{\hat{\delta}} R_{v} P_{\mathscr{M}_{2}} R_{v}^{*} L_{\hat{\delta}}^{*}=R_{v}\left(P_{\mathscr{M}_{2}}-L_{\hat{o}} P_{\mathbb{M}_{2}} L_{\hat{\delta}}^{*}\right) R_{v}^{*}=R_{v} P_{\mathscr{F}_{2}} R_{v}^{*}$. Thus

$$
v(x) P_{2}(x) v^{*}(x)=P_{1}(x)
$$

(a.e.). Since the multiplicity function $m_{i}(x)$ of $\mathscr{A}_{i}$ at the point $x$ is, by definition, the dimension of the range of $P_{i}(x)$ we have (a.e.)

$$
m_{1}(x)=\operatorname{rank} P_{1}(x)=\operatorname{rank} v(x) P_{2}(x) v^{*}(x) \leqq \operatorname{rank} P_{2}(x)=m_{2}(x) .
$$

To show that the second statement implies the third, we will assume that $X$ is a $\sigma$-compact separable metric space. Since we are already assuming that our measure spaces are standard Borel spaces, this is really no restriction at all. Let $B_{1}$ denote the unit ball of operators on $\ell^{2}(\boldsymbol{Z})$ equipped with the weak operator topology. The weak topology on any bounded weakly closed set of operators is the topology of a complete separable metric space [9, p. 38]. Let $\mathscr{Y}$ denote the set of partial isometries on $\ell^{2}(\boldsymbol{Z})$ and let $\mathscr{V}$ have the Borel structure induced by the weak operator topology. Recall (cf. [5]) that $\mathscr{V}=\left\{V \mid V \in B_{1}, V=V V^{*} V\right\}$. It follows as a corollary to Lemma 9.2 of [12] that $\mathscr{V}$ is a Borel subset of $B_{1}$.

Let $E=\left\{(x, v) \in X \times B_{1} \mid v \in \mathscr{Y}, v P_{2}(x) v^{*}=P_{1}(x)\right\}$. Then $E$ is a Borel (hence analytic) subset of $X \times B_{1}$. Furthermore $\left\{x \in X \mid\left(x, B_{1}\right) \cap\right.$ $E \neq \phi\}$ is almost all of $X$ due to the hypothesis that the multiplicity functions satisfy $m_{1}(x) \leqq m_{2}(x)$ (a.e.). Hence by von Neumann's 
principle of measurable choice $[9, \mathrm{p} .35]$, there exists a null set $N$ in $X$ and a Borel function $v: X \backslash N \rightarrow B_{1}$ such that $(x, v(x)) \in E$ for almost all $x$. Let $V=\int_{X}^{\oplus} v(x) d x$. Then $V P_{\mathscr{F}_{2}} V^{*}=P_{\mathscr{F}_{1}}$. Moreover $V$ is in $\mathfrak{M}_{L}^{\prime}$ and so $P_{\mathscr{F}_{1}} V$ is also in $\mathfrak{M}_{L}^{\prime}$. In addition $\left(V P_{\mathscr{F}_{2}}\right)\left(V P_{\mathscr{F}_{2}}\right)^{*}=$ $V P_{\mathscr{F}_{2}} V^{*}=P_{\mathscr{F}_{1}}$ and $\left(V P_{\mathscr{F}_{2}}\right)^{*}\left(V P_{\mathscr{F}_{2}}\right)=P_{\mathscr{F}_{2}} V^{*} V P_{\mathscr{F}_{2}} \leqq P_{\mathscr{F}_{2}}$. Thus $V P_{\mathscr{F}_{2}}$ is a partial isometry in $\mathfrak{M}_{L}^{\prime}$ with final space $\mathscr{F}_{1}$ and initial space contained in $\mathscr{F}_{2}$. Hence $P_{\mathscr{F}_{1}} \leqq P_{\mathscr{F}_{2}}$ in $\mathfrak{M}_{L}^{\prime}$.

To demonstrate the remaining implication suppose $W$ is a partial isometry in $\mathfrak{M}_{L}^{\prime}$ with the property that $W W^{*}=P_{\mathscr{F}_{1}}$ and $W^{*} W \leqq$ $P_{\mathscr{F}_{2}}$. We may write $L^{2}(\boldsymbol{Z} \times X)=\mathscr{H}_{1} \oplus \mathscr{C}_{2}$ where $\mathscr{C}_{1}=\sum_{n=-\infty}^{\infty} \oplus$ $L_{\delta}^{n} \mathscr{F}_{2}$ and $\mathscr{H}_{2}=\mathscr{H}_{1}^{\perp}$. The elements in $\mathscr{C}_{1}$ are sums $\sum_{n=-\infty}^{\infty} L_{i}^{n} e_{n}$ where $e_{n} \in \mathscr{F}_{2}$ and $\sum_{n=-\infty}^{\infty}\left\|L_{\hat{o}}^{n} e_{n}\right\|^{2}=\sum_{n=-\infty}^{\infty}\left\|e_{n}\right\|^{2}<\infty$. Define $\tilde{V}$ on $\mathscr{H}_{1}$ by the formula

$$
\widetilde{V}\left(\sum_{n=-\infty}^{\infty} L_{\hat{o}}^{n} e_{n}\right)=\sum_{n=-\infty}^{\infty} L_{\delta}^{n} W e_{n} .
$$

Extend $\tilde{V}$ to all of $L^{2}$ by defining $\tilde{V}\left(\mathscr{S} \mathscr{C}_{2}\right)=0$. Observe that $\tilde{V}$ is bounded because $W e_{n} \in \mathscr{F}_{1}$ and $\left\|\widetilde{V}\left(\sum L_{i}^{n} e_{n}\right)\right\|^{2}=\sum\left\|W e_{n}\right\|^{2} \leqq \sum\left\|e_{n}\right\|^{2}=$ $\left\|\sum L_{\hat{\delta}}^{n} e_{n}\right\|^{2}$. In fact once we show $\widetilde{V}$ commutes with $L_{\delta}$, the same computation shows that $\widetilde{V}$ is a partial isometry with initial space equal to $\sum_{n=-\infty}^{\infty} L_{i}^{n}\left(W^{*} W \mathscr{F}_{2}\right)$ and final space equal to $\sum_{n=-\infty}^{\infty} L_{\hat{0}}^{n} \mathscr{F}_{1}$. We now show that $\widetilde{V}$ commutes with $\&$.

$$
\begin{aligned}
\widetilde{V} L_{\hat{\delta}}\left(\sum L_{\hat{\delta}}^{n} e_{n}\right) & =\widetilde{V}\left(\sum L_{\hat{\delta}}^{n+1} e_{n}\right) \\
& =\sum L_{\hat{\delta}}^{n+1} W e_{n} \\
& =L_{\delta} \sum L_{\delta}^{n} W e_{n} \\
& =L_{\hat{o}} \widetilde{V}\left(\sum L_{\hat{o}}^{n} e_{n}\right) .
\end{aligned}
$$

And so $\widetilde{V}$ commutes with $L_{\dot{\delta}}$; finally, let $L_{\varphi}$ be in $\mathfrak{M}_{L}$.

$$
\begin{aligned}
\tilde{V} L_{\varphi}\left(\sum L_{\hat{o}}^{n} e_{n}\right) & =\widetilde{V}\left(\sum L_{\varphi} L_{\hat{o}}^{n} e_{n}\right) \\
& =\widetilde{V}\left(\sum L_{\hat{o}}^{n} L_{\varphi_{\circ \tau} n} e_{n}\right) \\
& =\sum L_{\hat{o}}^{n} W L_{\varphi_{\circ} n} e_{n} \\
& =\sum L_{\delta}^{n} L_{\varphi \circ \tau} W e_{n} \\
& =\sum L_{\varphi} L_{\hat{o}}^{n} W e_{n} \\
& =L_{\varphi}\left(\sum L_{\hat{o}}^{n} W e_{n}\right) \\
& =L_{\varphi} \widetilde{V}\left(\sum L_{\hat{\delta}}^{n} e_{n}\right) .
\end{aligned}
$$

Hence $\tilde{V}$ is in $\mathscr{L}^{\prime}$ which equals $\Re$; to indicate this we write $\widetilde{V}=R_{v}$. Next observe that $W P_{\mathscr{F}_{2}} W^{*}=P_{\mathscr{F}_{1}}$. To see this consider the partial isometry $W=W W^{*} W$. By hypothesis, $W^{*} W \leqq P_{\mathscr{F}_{2}}$ and so we have $W P_{\mathscr{F}_{2}} W^{*}=W W^{*} W P_{\mathscr{F}_{2}} W^{*}=W W^{*} W W^{*}=P_{\mathscr{F}_{1}}$. Using this fact we have 


$$
\begin{aligned}
R_{v} P{ }_{2} R_{v}^{*} & =R_{v} \sum_{n \leq 0} L_{\hat{o}}^{n} P_{\mathscr{F}_{2}} L_{\hat{o}}^{* n} R_{v}^{*} \\
& =\sum_{n \leq 0} L_{\hat{o}}^{n} R_{v} P_{\mathscr{F}_{2}} R_{v}^{*} L_{\hat{o}}^{*^{n}} \\
& =\sum_{n=0} L_{\hat{o}}^{n} W P_{F_{2}} W^{*} L_{\hat{o}}^{*^{n}} \\
& =\sum_{n \leq 0} L_{\hat{o}}^{n} P_{\mathscr{F}_{1}} L_{\hat{\delta}}^{* n} \\
& =P_{n_{1}} .
\end{aligned}
$$

Using the elementary properties of projections and partial isometries, it follows that $\mathscr{C}_{1}=R_{v} \mathscr{L}_{2}$. This completes the proof.

The following theorem is actually a corollary to the proof of Theorem 3.4. It relates the unitary equivalence classes of leftinvariant subspaces to equivalence classes of projections in $\mathfrak{M}_{L}^{\prime}$. It also shows that for each such equivalence class there is an essentially unique multiplicity function.

THeOREM 3.5. For $i=1,2$, let $\mathscr{L}_{i}, \mathscr{F}_{i}, P_{\mathscr{F}_{i}}$, and $m_{i}$ be as in Theorem 3.4. Assume, in addition, that $\mathscr{K}_{i}$ is a full subspace. The following statements are equivalent.

(1) $\mathscr{H}_{1}=R_{u} \mathscr{C l}_{2}$ for a unitary operator $R_{u}$ in $\Re$;

(2) $m_{1}(x)=m_{2}(x)$ (a.e.);

(3) $P_{F_{1}} \sim P_{\mathscr{F}_{2}}$ in $\mathfrak{M}_{L}^{\prime}$; and

(4) The algebras $\mathfrak{M}_{L} \mid \mathscr{F}_{1}$ and $\mathfrak{M}_{L} \mid \mathscr{F}_{2}$ are unitarily equivalent and the equivalence is implemented by a unitary operator in $\mathfrak{M}_{L}^{\prime}$.

Proof. The proof of the equivalence of the first three statements is similar to (and, in fact, easier than) that in 3.4. It should be noted that in case $R_{u}$ is unitary, the statements $\mathscr{M}_{1}=R_{u} \mathscr{C}_{2}$ and $P_{\mathscr{M}_{1}}=R_{u} P_{\mathscr{M}_{2}} R_{u}^{*}$ are equivalent. To show that the fourth assertion is equivalent to the other three we shall show that statement (1) implies (4) and then show that (4) implies (3).

Using the fact that $R_{u}$ is a unitary operator the following computation shows that $\mathscr{F}_{1}=R_{u} \mathscr{F}_{2}: \mathscr{F}_{1}=\mathscr{L}_{1} \ominus L_{\delta} \mathscr{M}_{1}=R_{u} \mathscr{M}_{2} \ominus L_{j} R_{u} \mathscr{K}_{2}=$ $R_{u} \mathscr{A}_{2} \ominus R_{u} L_{j} \mathscr{C l}_{2}=R_{u}\left(\mathscr{M}_{2} \ominus L_{j} \mathscr{H}_{2}\right)=R_{u} \mathscr{F}_{2}$. Clearly then the restriction $L_{\varphi} \mid \mathscr{F}_{1}$ equals $R_{u} L_{\varphi} \mid \mathscr{F}_{2} R_{u}^{*}$ for each $L_{\varphi}$ in $\mathfrak{M}_{L}$ and the result follows.

For the final implication, let $U$ be a unitary operator in $\mathfrak{M}_{L}^{\prime}$ such that $\mathfrak{M}_{L}\left|\mathscr{F}_{1}=U \mathfrak{M}_{L}\right| \mathscr{F}_{2} U^{*}$. Then $U \mathscr{F}_{2}=\mathscr{F}_{1}$ and $P_{\mathscr{F}_{1}} U$ is a partial isometry in $\mathfrak{M}_{L}^{\prime}$ with initial space $\mathscr{F}_{2}$ and final space $\mathscr{F}_{1}$. Hence $P_{\mathscr{F}_{1}}$ and $P_{\mathscr{F}_{2}}$ are equivalent projections in $\mathfrak{M}_{L}^{\prime}$.

The following example shows that there exist full pure $\&_{+}$ invariant subspaces that are not unitarily equivalent by a unitary in $\Re$. Because of the existence of such subspaces, it is impossible to represent all $\mathbb{S}_{+}$-invariant subspaces in the form $R_{v} \ell^{2}\left(\boldsymbol{Z}_{+}, L^{2}(X)\right)$ 
as in the generalization of Beurling's theorem in [6].

EXAMPLe 3.6. Let $X$ be the 3 point space: $X=\left\{x_{0}, x_{1}, x_{2}\right\}$. We shall treat $X$ as a measure space with counting measure. The transformation $\tau$ will be the cyclic permutation of $X$ defined by $\tau\left(x_{i}\right)=x_{i+1}(i=0,1), \tau\left(x_{2}\right)=x_{0}$. We shall write the Hilbert space $L^{2}(\boldsymbol{Z} \times X)$ as $\ell^{2}(\boldsymbol{Z} \times X)$ since everything in sight is discrete. Because $\tau$ is measure preserving, the Radon-Nikodym derivative $J_{n}$ is no longer necessary. For example, $\left(L_{\delta} f\right)(k, x)=f\left(k-1, \tau^{-1} x\right)$, for $f$ in $\ell^{2}(\boldsymbol{Z} \times X)$. If $C \leqq \boldsymbol{Z} \times X, \ell^{2}(C)$ will denote the subspace of $\ell^{2}(\boldsymbol{Z} \times X)$ consisting of those functions $f$ such that $f(n, x)=0$ for $(n, x) \notin C$. Finally recall that $\boldsymbol{Z}_{+}$is the set of nonnegative integers.

To construct subspaces that are not unitarily equivalent in $\Re$, let $B=\left(\boldsymbol{Z}_{+} \times X\right) \backslash\left\{\left(0, x_{0}\right)\right\}$ and let $\mathscr{C}_{1}=\ell^{2}\left(\boldsymbol{Z}_{+} \times X\right)$ and $\mathscr{C}_{2}=\ell^{2}(\boldsymbol{B})$. Clearly $\mathscr{C}_{1}$ and $\mathscr{L}_{2}$ are $\mathscr{Q}_{+}$-invariant subspaces and one can easily check that they are both pure and full. Finally, it is not difficult to show that $m_{1}$, the multiplicity function for the subspace $\mathscr{L}_{1}$, is the function $m_{1}\left(x_{i}\right)=1, i=0,1,2$. However the second subspace has multiplicity function $m_{2}$ with values $m_{2}\left(x_{0}\right)=0, m_{2}\left(x_{1}\right)=2, m_{2}\left(x_{2}\right)=$ 1. By Theorem 3.5, there is no unitary operator $R_{u}$ in $\Re$ such that $\mathscr{H}_{1}=R_{u} \mathscr{M}_{2}$.

4. Two-sided invariant subspaces. Following the motivation provided by Beurling's theorem, the next objective is to use the information from Theorem 3.5 to obtain a complete set of parameters for labeling the invariant subspaces. At this level of generality this is a very difficult problem. The next theorem shows that when the transformation $\tau$ is also assumed to be ergodic and freely acting, the two-sided invariant subspaces are parameterized very conveniently in terms of certain subsets of $Z \times X$.

In this section we shall assume that the transformation $\tau$ generates an infinite group, thus excluding periodic transformations. We shall also assume that both $\tau$ and $\tau^{-1}$ are ergodic transformations so that if $\tau(E) \subseteq E$ and $\tau^{-1}(E) \subseteq E$ for some measurable set $E$ in $X$ then either $E$ or its complement $X \backslash E$ is a set of measure zero. This is equivalent to the assumption that the group generated by $\tau$ is an ergodic group [7, p. 195]. It should be noted [1, p. 596] that ergodicity implies that the group generated by $\tau$ is freely acting in the sense that no (nonzero) power of $\tau$ is the identity on any piece of $X$ of positive measure. The formal definition is that for every integer $n \neq 0$ and every measurable set $E$ of positive measure, there exists a measurable subset $E_{0} \subseteq E$ such that $\tau^{n}\left(E_{0}\right) \cap$ $E_{0}=\phi$ and $\mu\left(E_{0}\right)>0$. It is well known that $\&$ and $\Re$ are factors 
when $\tau$ is ergodic (and freely acting) [8].

We shall need the following notation. If $T$ is any bounded linear operator on $L^{2}(\boldsymbol{Z} \times X)$, we can decompose $T$ into an infinite (operator) matrix $\left[T_{n k}\right]$ where $T_{n k}$ is defined by

$$
(T f)(n, x)=\left(\sum_{k=-\infty}^{\infty} T_{n k} f(k, \cdot)\right)(x), \quad f \in L^{2} ;
$$

each $T_{n k}$ is a bounded operator on $L^{2}(X)$. For example, if we let $I$ denote the identity operator on $L^{2}(X)$, then the operator $R_{\delta}$ has as its associated matrix

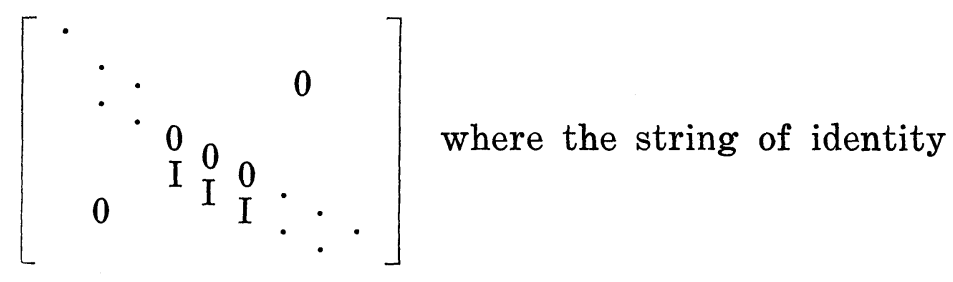

operators is on the first subdiagonal. We shall let $\mathfrak{M}$ denote the weakly closed algebra $\mathfrak{M}_{L} \vee \mathfrak{M}_{R}$ generated by $\mathfrak{M}_{L}$ and $\mathfrak{M}_{R}$. In the description of the invariant subspaces for the algebra $\mathfrak{L}_{+} \vee \Re_{+}$, we shall need to know the form of the operators in the commutant of $\mathfrak{M}$. This form has been computed by Singer in [10] under the assumption that $X$ is a finite measure space and $\tau$ is measure preserving. Elementary modifications of his work show that the following results are valid in our setting as well.

Proposition 4.1 (cf. [10, Lemma 2.4]). If $T$ is an operator in $\mathfrak{M}^{\prime}$, the commutant of $\mathfrak{M}$, with associated matrix $\left[T_{i j}\right]$, then

$$
T_{i j}= \begin{cases}L_{\varphi_{j}}, & i=j \text { where } \varphi_{j} \in L^{\infty}(X), \\ 0, & i \neq j\end{cases}
$$

CoROLlaRY 4.2 (cf. [10, Corollary 2.4]). The algebra $\mathfrak{M}=\mathfrak{M}_{L} \vee$ $\mathfrak{M}_{R}$ is a maximal abelian selfadjoint algebra in $\mathscr{L}\left(L^{2}(\boldsymbol{Z} \times X)\right.$ ), the bounded operators on $L^{2}$.

The theorem we are about to state shows that there is a oneto-one correspondence between two-sided invariant subspaces and measurable sets in $\boldsymbol{Z} \times X$ which are invariant for two very simple maps on $\boldsymbol{Z} \times X$. These maps, $\lambda$ and $\rho$, are defined as follows:

$$
\lambda(n, x)=(n+1, \tau x)
$$




$$
\rho(n, x)=(n+1, x)
$$

where, recall, $\tau$ is the invertible ergodic measurable transformation on the space $X$ with quasi-invariant measure $\mu$. Although we shall state Theorem 4.3 with the assumption that $\tau$ has no wandering set, this condition is not necessary. In fact if $\tau$ has a wandering set then the $\sigma$-algebra for the measure space $X$ must be atomic and $X$ then consists of a countable number of atoms. The space $L^{2}(\boldsymbol{Z} \times X)$ becomes essentially $\ell^{2}(\boldsymbol{Z} \times \boldsymbol{Z})$ and the theorem can be proved in this case also.

THEOREM 4.3. Let $\tau$ be as described at the beginning of this section and assume also that $\tau$ has no wandering sets. The subspace th is invariant for $\mathbb{L}_{+} \vee \Re_{+}$if and only if $\mathscr{l}=L^{2}(B)$ for some subset $B$ of $\boldsymbol{Z} \times X$ which is invariant for both $\lambda$ and $\rho$.

Proof. Observe that the theorem is trivial in case $\mathscr{C}=$ $L^{2}(\boldsymbol{Z} \times X)$ or $\mathscr{C}=\{0\}$. Therefore we shall assume $\mathscr{C}$ is a nontrivial subspace and produce the set $B$. The first and most important step in this portion of the proof is to show that the subspace $\mathscr{C l}_{\mathrm{C}}$ is right-pure. Using the right-handed version of Proposition 3.3, we may write $\mathscr{C}_{\mathscr{C}}=\mathscr{C}_{1} \oplus \mathscr{C}_{2}$ where $\mathscr{C}_{1}$ is right-reducing and $\mathscr{C}_{2}$ is right-pure. Since $\mathscr{C}_{1}=\bigcap_{n 0} R_{i}^{n} \mathscr{C}$, it is clear that $\mathscr{C}_{1}$ is invariant under $\mathbb{S}_{+} \vee \Re_{+}$. We show that $\mathscr{C}_{1}$ is the zero subspace and hence $\mathscr{C}=\mathscr{C}_{2}$ is right-pure. Since $\mathscr{C}_{1}$ is right-reducing,

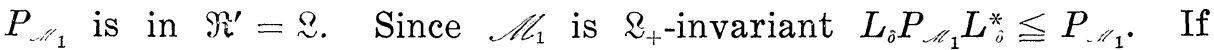
equality holds, then $P_{\mathscr{N}_{1}}$ commutes with $L_{\hat{o}}$ and hence $P_{\|_{1}}$ is in $\Re^{\prime} \cap \mathbb{S}^{\prime}=(\mathfrak{R} \vee \mathfrak{Z})^{\prime}=\{\boldsymbol{C I}\}$, the scalar multiples of the identity. Since $\mathscr{C}_{1}$ is not all of $L^{2}(\boldsymbol{Z} \times X)$ by hypothesis, $\mathscr{C}_{1}$ must be the zero subspace.

Before considering the case that $L_{\hat{o}} P_{\mathbb{N}_{1}} L_{\hat{o}}^{*} \lesseqgtr P_{\mathbb{M}_{1}}$, we show that $P_{\mathscr{M}_{1}}=L_{1_{E}}$ for some indicator function $1_{E}$ on $X$. Since $\mathscr{C}_{1}$ is $\mathfrak{L}_{+} \vee$ $\mathfrak{R}_{+}$-invariant, $P_{\mathscr{N}_{1}}$ is in $\mathfrak{M N}_{L}^{\prime} \cap \mathfrak{M}_{R}^{\prime}=\mathfrak{M}^{\prime}$, by Proposition 3.1. By Proposition $4.1, P_{\mathbb{N}_{1}}$ has a matrix representation $\left[P_{i j}\right]$ where $P_{i j}=0$ for $i \neq j$ and $P_{j j}$ is multiplication by an $L^{\infty}$-function for each $j$. Since $P_{\mathscr{N}_{1}}$ is a projection, it follows that $P_{j j}$ is multiplication by an indicator function $1_{E_{j}}$ on $X$. Thus $\left(P_{\mathscr{N}_{1}} f\right)(n, x)=1_{E_{n}}(x) f(n, x)$ for $f$ in $L^{2}$. But since $\mathscr{C}_{1}$ reduces $\Re, P_{\mathscr{M}_{1}}$ commutes with $R_{j}$. Hence for any $f$ in $L^{2}$ and integers $n$ and $m, 1_{E_{n}}(x) f(n-m, x)=\left(P_{M_{1}} R_{\dot{o}}^{m} f\right)(n, x)=$ $\left(R_{\bar{o}}^{m} P_{\mathscr{M}_{1}} f\right)(n, x)=1_{E_{n-m}}(x) f(n-m, x)$ (a.e.). From this it follows that $E_{n}=E_{m}$ for all $n$ and $m$ (up to a null set) and so $P_{" 11}=L_{1_{F}}$ with $E=E_{n}$.

Now consider the case that $L_{\hat{o}} P_{\mathscr{M}_{1}} L_{0}^{*} \varsubsetneqq P_{\mathscr{N}_{1}}$. We have $L_{1^{*}}=$ $P_{\mathscr{A}_{1}}>L_{\hat{o}} P_{\|_{1}} L_{i}^{*}=L_{\hat{o}} L_{1_{i j}} L_{\hat{o}}^{*}=L_{1_{L^{*} \tau^{-1}}}=L_{1_{\tau}(E)}$. Thus $m(E \backslash \tau(E))>0$. 
But $E \backslash \tau(E)$ is a wandering subset (of positive measure) contradicting our hypothesis. So we conclude that $\mathscr{K}_{1}=\{0\}$ and $\mathscr{K}$ is right-pure.

Let $\mathscr{F}=\mathscr{C} \Theta R_{\delta} \mathscr{C}$ be the (right) wandering subspace for $R_{\delta}$ and let $P_{\mathscr{F}}$ be the projection onto $\mathscr{F}$. The right-handed version of Lemma 3.2 shows that $P_{\mathscr{G}}$ is in $\mathfrak{M}_{R}^{\prime}$. Since $\mathscr{C}$ and $R_{\delta} \mathscr{C}$ are both $\mathscr{L}_{+}$-invariant (and hence reduce $\mathfrak{M}_{L}$ by 3.1 ), $\mathscr{F}=\mathscr{C} \cap\left(R_{\tilde{\delta}} \mathscr{C l}\right)^{\perp}$ reduces $\mathfrak{M}_{L}$ so that $P_{\mathscr{F}}$ is in $\mathfrak{M}_{L}^{\prime}$. Thus $P_{\mathscr{F}}$ lies in $\mathfrak{M}^{\prime}$. By Proposition 4.1 and the remarks in the preceding paragraphs, there exists a sequence of sets $\left\{E_{n}\right\}$ in $X$ such that $\left(P_{\mathscr{F}} f\right)(n, x)=1_{E_{n}}(x) f(n, x)$ (a.e.) for $f$ in $L^{2}$.

We now show that $\mathscr{M}=L^{2}(B)$ where $B=\bigcup_{n=-\infty}^{\infty} \boldsymbol{Z}_{(n)} \times E_{n}$ with $\boldsymbol{Z}_{(n)}$ equal to the set $\{n, n+1, n+2, \cdots\}$. Clearly $\mathscr{F}=\sum_{n=-\infty}^{\infty} \oplus$ $L^{2}\left(\{n\} \times E_{n}\right)$. This is a direct sum since $\left(\{n\} \times E_{n}\right) \cap\left(\{m\} \times E_{m}\right)=$ $\phi, n \neq m$. Since $\mathscr{C}$ is a pure subspace, we have

$$
\begin{aligned}
\mathscr{M} & =\sum_{k=0}^{\infty} \oplus R_{i}^{k} \mathscr{F} \\
& =\sum_{k=0}^{\infty} \oplus R_{\hat{o}}^{k}\left(\sum_{n=-0}^{\infty} L^{2}\left(\{n\} \times E_{n}\right)\right) \\
& =\sum_{n=-\infty}^{\infty} \oplus\left(\sum_{k=0}^{\infty} R_{\delta}^{k} L^{2}\left(\{n\} \times E_{n}\right)\right) \\
& =\sum_{n=-\infty}^{\infty} \oplus\left(L^{2}\left(\{n\} \times E_{n}\right) \oplus L^{2}\left(\{n+1\} \times E_{n}\right) \oplus \cdots\right) \\
& =\sum_{n=-\infty}^{\infty} \oplus L^{2}\left(\boldsymbol{Z}_{(n)} \times E_{n}\right) \\
& =L^{2}(B) .
\end{aligned}
$$

Note that $B$ is clearly invariant for $\rho$. To see that $B$ is invariant for $\lambda$, let $(n, x)$ be in $B$. Observe that $\lambda(n, x) \in B$ if there is an element in the subspace $\mathscr{C}$ whose support contains $\lambda(n, x)$. Let $f$ be a function in $\mathscr{C}$ whose support contains $(n, x)$. Then $L_{\hat{o}} f$ is in $\mathscr{M}$ and $\left(L_{\delta} f\right)(\lambda(n, x))=\left(L_{\delta} f\right)(n+1, \tau x)=J_{1}^{1 / 2}(\tau x) f(n, x) \neq 0$ (a.e.) since $J_{1}$ is nonzero (a.e.).

To complete the proof, let $\mathscr{C}=L^{2}(B)$ for some set $B \leqq Z \times X$ invariant for $\lambda$ and $\rho$. We need to show that supp $T f$, the support of $T f$, is contained in $B$ for any $T$ in $\mathfrak{L}_{+} \vee \Re_{+}$and $f$ in $L^{2}(B)$. This is clear for $T$ in $\mathfrak{M}$ so we need only show that the supports of $L_{\delta} f$ and $R_{\delta} f$ are contained in $B$. Suppose $(n, x)$ is an element of the support of $L_{\delta} f$ so that $0 \neq\left(L_{\delta} f\right)(n, x)=J_{1}^{1 / 2}(x) f\left(n-1, \tau^{-1} x\right)=$ $J_{1}^{1 / 2}(x) f\left(\lambda^{-1}(n, x)\right)$. Thus $f\left(\lambda^{-1}(n, x)\right) \neq 0$ so that $\lambda^{-1}(n, x)$ is in $B$. But this implies $(n, x)$ is in $B$ by invariance of $B$ for the map $\lambda$. Similarly, the support of $R_{\delta} f$ is contained in $B$.

REMARK 4.1. A nearly identical "left-hand" proof can be con- 
structed by showing that $\mathscr{C}$ is left-pure in case $\tau$ has no wandering sets. The same proof allows us to obtain the pure invariant subspaces for the algebras $\Re_{+} \vee \mathfrak{M}_{L}$ and $\mathfrak{L}_{+} \vee \mathfrak{M}_{R}$. These subspaces are of the form $L^{2}(B)$ where $B$ is $\rho$-invariant in the case of the algebra $\mathfrak{R}_{+} \vee \mathfrak{M}_{L}$ and $\lambda$-invariant in the other case.

(2) In the proof of Theorem 4.3 we produced a sequence of sets $\left\{E_{n}\right\}_{n=-\infty}^{\infty}$. Using the definition of $R_{\delta}$ and the fact that the subspace $\mathscr{F}$ is wandering for $R_{\delta}$ it follows that the sets $\left\{E_{n}\right\}$ are pairwise disjoint (up to a null set). In case the union $\cup_{n \in Z} E_{n}$ is almost all of $X$, the subspace in question is not only right-pure but also right-full. This is a consequence of Proposition 3.1.

(3) It can also be shown as a consequence of ergodicity that when $X$ is a space with a finite invariant measure, the sequence $\left\{E_{n}\right\}$ forms a partition of $X$. Thus two-sided invariant subspaces in this case are both full and pure. In addition, under these hypotheses on $X$, an alternate and illuminating proof of this last fact can be given.

Proposition 4.5. If $\mu$ is a finite invariant measure on $X$, then any nontrivial $\mathfrak{Q}_{+} \vee \Re_{+}$-invariant subspace is both (left) full and pure.

Proof. Observe first that under the current assumptions on $\mu$ and $\tau$, the algebras $\&$ and $\Re$ are finite factors. Decompose $\mathscr{C}$ into $\mathscr{M}_{1} \oplus \mathscr{C}_{2}$ as in Proposition 3.3. To show $\mathscr{C}$ is pure we need to show $\mathscr{C}_{1}=\{0\}$. Let $P$ be the projection onto $\mathscr{C}_{1}$ and observe that $P$ is in $\mathscr{L}^{\prime}$ since $\mathscr{M}_{1}$ is left-reducing.

We assert also that $P$ is in $\&$. To prove this we show $P \in$ $\Re^{\prime}(=\mathbb{R})$. Clearly $P$ commutes with $\mathfrak{M}_{R}$ and so we need only show that $P$ commutes with $R_{\delta}$. Note that $R_{\delta} P R_{\delta}^{*}$ is the projection onto the subspace $\Re_{+} \mathscr{M}_{1}=\Re_{+} P L^{2}$. By the right-invariance of $\mathscr{C}$ and since $\Re_{+} \subseteq R^{\prime}, \Re_{+} P L^{2} \subseteq P L^{2}$. Hence the projection $R_{\delta} P R_{\delta}^{*}$ is dominated by $P$. But $R_{\delta} P R_{\delta}^{*}$ and $P$ are equivalent projections in $\Re$. (In fact, if we let $U=R_{\delta} P$, then $U$ is a partial isometry in $\Re$, $U U^{*}=R_{\delta} P R_{\delta}^{*}$, and $U^{*} U=P$.) Since $\Re$ is a finite von Neumann algebra, it follows that $R_{\delta} P R_{\delta}^{*}=P$. This shows that $P$ commutes with $\Re$ and hence lies in $\mathbb{R}$. Thus $P$ is in $\mathbb{R} \cap \mathbb{R}^{\prime}$, the center of the algebra $\&$. Since $\&$ is a factor, $P$ must be either the zero operator or the identity. Since $\mathscr{M}$ is not $L^{2}, P \neq I$ and so $P=0$. Hence $\mathscr{A}_{1}$ is the zero subspace.

To prove $\mathscr{M}$ is full, let $\mathscr{N}=\mathrm{V}_{n \leqq 0} L_{\delta}^{n} \not \mathscr{C}$. A proof nearly identical to the one above shows that the projection onto $\mathscr{N}$ lies in the center of $\mathbb{R}$. Since $\mathscr{N} \neq\{0\}$, we must have $\mathscr{N}=L^{2}(Z \times X)$ and hence $\mathscr{H}$ is full. 
5. An example. In this section an example will be given to show how Theorem 4.3 can fail when the transformation $\tau$ on $X$ is periodic. For this example let $X$ be the space consisting of $n$ points $\left\{x_{0}, x_{1}, \cdots, x_{n-1}\right\}$ for $n \geqq 2$. Just as in Example 3.6 where $n=3$, let $\tau$ be the permutation of $X$ defined by $\tau\left(x_{i}\right)=x_{i+1}(i \neq n-1)$ and $\tau\left(x_{n-1}\right)=x_{0}$. Observe that since $\tau$ is periodic we have

$$
\left(L_{\hat{o}}^{n} f\right)(k, x)=f\left(k-n, \tau^{-n} x\right)=f(k-n, x)=\left(R_{\tilde{\delta}}^{n} f\right)(k, x)
$$

for $f$ in $\ell^{2}(\boldsymbol{Z} \times X)$, so that $L_{\hat{\delta}}^{n}=R_{\dot{\delta}}^{n}$. In particular $L_{\dot{\delta}}^{n}$ is in the center of $\mathcal{L}$ (and $\mathfrak{R}$ ) and hence $\mathcal{L}$ and $\Re$ are not factors as in the previous section.

To show that Theorem 4.3 fails in this situation, we shall construct a subspace $\mathscr{C}$ contained in $\ell^{2}(\boldsymbol{Z} \times X)$ such that $\mathscr{C}$ is invariant for the algebra $\mathscr{S}_{+} \vee \Re_{+}$but $\mathscr{C}$ is not of the form $\ell^{2}(B)$ for any subset $B$ of $Z \times X$. (Observe that the other half of 4.3 is always valid. Namely, if $B$ is invariant under the transformations $\lambda$ and $\rho$, then $\ell^{2}(B)$ is $\mathfrak{L}_{+} \vee \Re_{+}$-invariant.)

Let $\theta$ be a finite Blaschke product with zeros $\left\{\alpha_{1}, \cdots, \alpha_{k}\right\}$ in the punctured unit disk (i.e., $0<\left|\alpha_{i}\right|<1, i=1,2, \cdots, k$ ); thus $\theta$ has the form $\theta(z)=\prod_{n=1}^{k}\left(\left|\alpha_{n}\right| / \alpha\right)_{n}\left(\alpha_{n}-z\right) /\left(1-\bar{\alpha}_{n} z\right)$. Let $L_{n}=\theta\left(L_{i}^{n}\right)$ be the unitary operator defined by $\theta$ and the operator $L_{\hat{o}}^{n}$ via the functional calculus. Let $\sum_{k=0}^{\infty} a_{k} z^{k}$ be the power series for $\theta$. Since the power series converges absolutely, the series $\sum_{k=0}^{\infty} a_{k}\left(L_{\delta}^{n}\right)^{k}$ converges in norm to the operator $L_{u}$. Observe that $a_{0}$ is not zero since the function $\theta$ does not vanish at the origin. Observe also that since $L_{o}^{n}$ is in the center of $\mathbb{2}_{+}$, it follows, again by the functional calculus, that $L_{u}$ is in the center of $\Omega_{+}$. Define the subspace $\mathscr{C}$ to be $L_{u} \ell^{2}\left(\boldsymbol{Z}_{+} \times X\right)$. Since $L_{u}$ is in the center and $\ell^{2}\left(\boldsymbol{Z}_{+} \times X\right)$ is a two-sided invariant subspace, $\mathscr{C}$ is also a two-sided invariant subspace. We shall show that $\mathscr{C} \neq \ell^{2}(B)$ for any set $B$.

For any subspace $\mathscr{N} \subseteq \ell^{2}(Z \times X)$, let $E_{0}^{\mathscr{r}}=U\{x \in X \mid f(0, x) \neq 0\}$. Then $E_{0}{ }^{r}$ is the support set $\operatorname{supp}\{f(0, \cdot) \mid f \in \mathscr{N}\}$. In case the subspace is $\ell^{2}\left(Z_{+} \times X\right)$, this support set is all of $X$. Since

$$
\begin{aligned}
\left(L_{u} f\right)(0, x) & =\sum_{k=0}^{\infty} a_{k}\left(L_{\delta}^{n k} f\right)(0, x)=\sum_{k=0}^{\infty} a_{k} f\left(-n k, \tau^{-n k} x\right) \\
& =a_{0} f(0, x)
\end{aligned}
$$

for any function $f$ in $\ell^{2}\left(\boldsymbol{Z}_{+} \times X\right)$, it follows that $E_{0} "=X$ also. Now if $\mathscr{C}=\ell^{2}(B)$ (for some $B \subseteq Z \times X$ ), we must have $B=Z_{+} \times X$ since $\mathscr{C}$ is a two-sided invariant subspace (and hence $B$ must be $\lambda$ and $\rho$ invariant). Thus $\ell^{2}\left(\boldsymbol{Z}_{+} \times X\right)=L_{u} \ell^{2}\left(\boldsymbol{Z}_{+} \times X\right)$. Since $L_{u}$ is unitary, we must also have $L_{u}^{*} \ell^{2}\left(\boldsymbol{Z}_{+} \times X\right)=\ell^{2}\left(\boldsymbol{Z}_{+} \times X\right)$ which is clearly impossible for the unitary operator $L_{u}$ constructed above. 
Hence $\mathscr{l l} \neq \ell^{2}(B)$ for any $B \subseteq Z \times X$.

To obtain more examples, perhaps an exhaustive collection, we note that with appropriate modifications, the finite Blaschke product could be replaced by any nonconstant inner function $\theta$ with nonvanishing zeroth Fourier coefficient. Additionally, the subspace $\ell^{2}\left(\boldsymbol{Z}_{+} \times X\right)$ could be replaced by certain other subspaces of the form $\ell^{2}(C)$. Again the subspace $\theta\left(L_{i}^{n}\right) \ell^{2}(C)$ would not have the form $\ell^{2}(B)$ for a subset $B \subseteq Z \times X$.

6. Concluding remarks. An interesting feature of the algebras we have been considering is that they can be developed from several different points of view. For example, in $\S 2$ of [6] it is shown how the algebras $\&$ and $\Re$ may be represented as the left and right von Neumann algebras associated with a Hilbert algebra. (In fact it is this representation that motivates our notation here.) In $\S$ I.9.2 of [3], Dixmier introduces crossed products using a matricial construction. We show that the algebras studied here can be similarly represented. As a corollary, it is easily seen that $\mathbb{S}_{+}$and $\mathfrak{R}_{+}$are algebras of lower triangular matrices.

In this section we assume that $X$ is a finite measure space and $\tau$ is measure preserving. The transformation $U$ defined by the equation $(U f)(x)=f(\tau x)$ is a unitary operator on $L^{2}(X)$. This operator normalizes $L^{\infty}$, the algebra of multiplication operators on $L^{2}(X)$. In the following proposition, we use the term "crossed product" to refer to the algebras constructed by Dixmier in [3, $\S[.9 .2]$.

Proposition 6.1. The algebras $\&$ and $\Re$ are the left and right crossed products determined by $L^{\infty}(X)$ and (the group of automorphisms generated by) $U$.

Proof. As noted earlier, $L^{2}(\boldsymbol{Z} \times X)$ may be identified with $\sum_{-\infty}^{\infty} \oplus L^{2}(X)$. This identification is implemented by the map $W$ which takes a function $f$ in $L^{2}(Z \times X)$ to the sequence of functions $\left\{g_{n}\right\}_{n \in \boldsymbol{Z}}$ where $g_{n}(x)=f(n, x)$. It is convenient to write $g(n)$ for $g_{n}$. Using this notation we have $\left(W L_{\hat{o}} W^{*} g\right)(n)=U g(n-1)$. Thus the matrix representation for $W L_{\hat{o}} W^{*}$ is

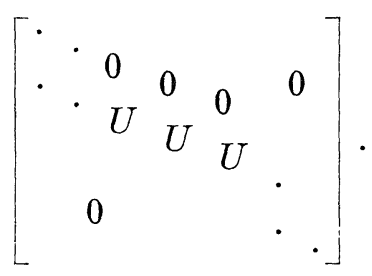


Likewise $\left(W L_{\varphi} W^{*} g\right)(n)=\varphi \cdot g(n)$ so that the associated matrix is

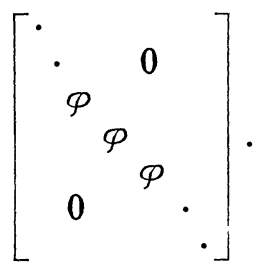

(In the notation of [3], $W L_{\delta} W^{*}=\widetilde{U}_{1}$ and $W L_{\varphi} W^{*}=\Phi(\varphi)$.) It is now clear that $W \& W^{*}$ is the crossed product of $L^{\infty}(X)$ and $U$. The description of $\Re$ is, of course, similar and is left to the reader.

\section{REFERENCES}

1. Wm. B. Arveson, Analyticity in operator algebras, Amer. J. Math., 89 (1967), $578-642$.

2. - Operator algebras and measure preserving automorphisms, Acta. Math., 118 (1967), 95-109.

3. J. Dixmier, Let algə̀bras d'opérateurs dans l'espace Hilbertien, Gauthier-Villars, Paris, 1969.

4. J. Feldman and C. Moore, Ergodic equivalence relations, cohomology, and von Neumann algebras II, Trans. Amer. Math. Soc., 234 (1977), 325-359.

5. P. R. Halmos, A Hilbert Space Problem Book, van Nostrand-Reinhold, Princeton, 1967.

6. M. McAsey, P. S. Muhly, and K.-S. Saito, Non-self-adjoint crossed products, Trans. Amer. Math. Soc., 248 (1979), 381-409.

7. F. J. Murray and J. von Neumann, On rings of operators, Ann. of Math., 37 (1936), 116-229.

8. J. von Neumann, On rings of operators III, Ann. of Math., 41 (1940), 94-161.

9. J. T. Schwartz, $W^{*}$-algebras, Gordon and Breach, New York, 1967.

10. I. M. Singer, Ausomorphisms of finite factors, Amer. J. Math., 77 (1955), 117-133.

11. M. Takesaki, Duality for crossed products and the structure of von Neumann algebras of type III, Acta Math., 131 (1973), 249-310.

12. V. S. Varadarajan, Geometry of Quantum Theory, Vol. II, van Nostrand-Reinhold, New York, 1970.

Received January 19, 1979 and in revised form April 1, 1980. This paper is a portion of the author's doctoral dissertation written at the University of Iowa under the supervision of Paul S. Muhly.

BRADLEY UNIVERSITY

PeORIA, IL 61625

Editorial: The preceding paper was to have been published prior to its sequel "Canonical Models for Invariant Subspaces" (this Journal 91 (1980) 377-395). The Pacific Journal inadvertently switched the order of publication. In the 1980 article, reference 5 is the current paper, while reference 6 in both papers should be as above. 



\section{PACIFIC JOURNAL OF MATHEMATICS}

\section{EDITORS}

DoNALD BABBITT (Managing Editor)

University of California

Los Angeles, CA 90024

Hugo Rossi

University of Utah

Salt Lake City, UT 84112

C. C. MOORE and ANDREw OGG

University of California

Berkeley, CA 94720
J. DugundJI

Department of Mathematics

University of Southern California

Los Angeles, CA 90007

R. FinN and J. Milgram

Stanford University

Stanford, CA 94305

ASSOCIATE EDITORS
R. ARENS
E. F. BECKENBACH
B. H. NeumanN
F. WOLF
K. YoshidA

\section{SUPPORTING INSTITUTIONS}

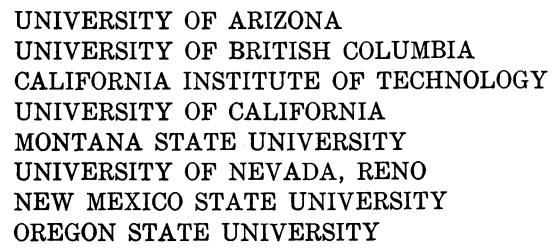

UNIVERSITY OF ARIZONA

UNIVERSITY OF BRITISH COLUMBIA

CALIFORNIA INSTITUTE OF TECHNOLOGY

UNIVERSITY OF CALIFORNIA

MONTANA STATE UNIVERSITY

UNIVERSITY OF NEVADA, RENO

NEW MEXICO STATE UNIVERSITY OREGON STATE UNIVERSITY

\author{
UNIVERSITY OF OREGON \\ UNIVERSITY OF SOUTHERN CALIFORNIA \\ STANFORD UNIVERSITY \\ UNIVERSITY OF HAWAII \\ UNIVERSITY OF TOKYO \\ UNIVERSITY OF UTAH \\ WASHINGTON STATE UNIVERSITY \\ UNIVERSITY OF WASHINGTON
}

The Supporting Institutions listed above contribute to the cost of publication of this Journal, but they are not owners or publishers and have no responsibility for its content or policies.

Mathematical papers intended for publication in the Pacific Journal of Mathematics should be in typed form or offset-reproduced, (not dittoed), double spaced with large margins. Please do not use built up fractions in the text of the manuscript. However, you may use them in the displayed equations. Underline Greek letters in red, German in green, and script in blue. The first paragraph or two must be capable of being used separately as a synopsis of the entire paper. Please propose a heading for the odd numbered pages of less than 35 characters. Manuscripts, in triplicate, may be sent to any one of the editors. Please classify according to the scheme of Math. Reviews, Index to Vol. 39. Supply name and address of author to whom proofs should be sent. All other communications should be addressed to the managing editor, or Elaine Barth, University of California, Los Angeles, California, 90024.

50 reprints to each author are provided free for each article, only if page charges have been substantially paid. Additional copies may be obtained at cost in multiples of 50 .

The Pacific Journal of Mathematics is issued monthly as of January 1966. Regular subscription rate: $\$ 102.00$ a year (6 Vols., 12 issues). Special rate: $\$ 51.00$ a year to individual members of supporting institutions.

Subscriptions, orders for numbers issued in the last three calendar years, and changes of address shoud be sent to Pacific Journal of Mathematics, P.O. Box 969, Carmel Valley, CA 93924, U.S.A. Old back numbers obtainable from Kraus Periodicals Co., Route 100, Millwood, NY 10546.

\footnotetext{
PUBLISHED BY PACIFIC JOURNAL OF MATHEMATICS, A NON-PROFIT CORPORATION

Printed at Kokusai Bunken Insatsusha (International Academic Printing Co., Ltd.). 8-8, 3-chome, Takadanobaba, Shinjuku-ku, Tokyo 160, Japan.
} 


\section{Pacific Journal of Mathematics}

\section{Vol. 96, No. 2 December, 1981}

Gerald A. Beer, A natural topology for upper semicontinuous functions and

a Baire category dual for convergence in measure $\ldots \ldots \ldots \ldots \ldots \ldots 251$

Georgia Benkart and J. Marshall Osborn, An investigation of real

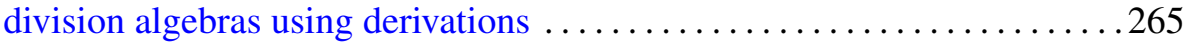

Donald Ian Cartwright and John R. McMullen, A structural criterion for the existence of infinite Sidon sets ........................ 301

Philip Hanlon, The fixed-point partition lattices $\ldots \ldots \ldots \ldots \ldots \ldots \ldots \ldots$

Eric Hayashi, The spectral density of a strongly mixing stationary Gaussian process

Chung-Wu Ho and Charles E. Morris, Jr., A graph-theoretic proof of

Sharkovsky's theorem on the periodic points of continuous functions . ...361

Sara Hurvitz, The automorphism groups of spaces and fibrations ....... 371

Atsushi Inoue, Schoichi Ota and Jun Tomiyama, Derivations of operator algebras into spaces of unbounded operators . .................. 389

Wolfgang B. Jurkat and Gary Sampson, On weak restricted estimates and endpoint problems for convolutions with oscillating kernels. I ........ 405

Georgios Koumoullis, Some topological properties of spaces of

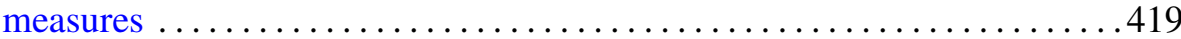

Wen Hsiung Lin, Algebraic Kahn-Priddy theorem ................. 435

Michael John McAsey, Invariant subspaces of nonselfadjoint crossed

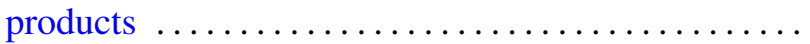

Justin Peters, Entropy of automorphisms on L.C.A. groups

Saburou Saitoh, A characterization of the adjoint $L$-kernel of Szegó type 